\title{
Advanced Optical Imaging of Blood Thrombus
}

\author{
Xuefei He ${ }^{l}$, Samantha J. Montague ${ }^{1,2}$, Xu Tao ${ }^{1}$, Elizabeth E. Gardiner ${ }^{1}$, Woei Ming Lee 1,2,3,* \\ ${ }^{1}$ Research School of Electrical, Energy and Materials Engineering,, College of Engineering and Computer Science, The \\ Australian National University, Canberra ACT 2601, Australia \\ ${ }^{2}$ ACRF Department of Cancer Biology and Therapeutics, John Curtin School of Medical Research, The Australian National \\ University, Canberra ACT 2601, Australia \\ ${ }^{3}$ The ARC Centre of Excellence in Advanced Molecular Imaging, The Australian National University, ACT 2601 Australia
}

\begin{abstract}
The application of advanced optical imaging technology for platelet biology is in its infancy. In this talk, we shall introduce the potential impact of optical imaging in understanding the roles of platelets in thrombus formation and in hemostasis. We will summarize how techniques in optical imaging has enabled exploration of morphological, molecular and fluidic parameters that determine platelet adhesion, activation and consequent roles in thrombus formation. Finally, we discuss the convergence of multiple imaging modalities towards a complete understanding of platelet roles in thrombus formation.
\end{abstract}

\section{Thrombus formatin}

Discoveries in biological sciences have largely been driven by the advances in technological tools. The first observation of platelets were made by Max Schultze who used a 17th century optical microscope with a moist chamber to reveal individual granulates (particles) during the process of coagulation (formation of a blood clot). However, this method was insufficient for in depth investigations. It was only until the use of immersion microscope objective by Bizzozero, an Italian microscopist, in 1881 that lead to an in-depth characterization of platelets as an independent component of blood cells around the mesenteric vessels of rabbits and guinea pigs. A key finding by Bizzozero was the crucial role of platelets in the formation of hemostatic thrombus following a controlled injury to blood vessels.

Since then, platelets have been established as a crucial component of blood due to their fundamental roles in hemostasis (process of stopping blood flow). Studies of platelets are generally focused on deeper understanding of the finely regulated molecular mechanism in hemostasis and thrombus formation (process of blood clot), which have major involvement in o different diseases progressions such as atherosclerosis, angiogenesis and tumorigenesis. Recently, platelets have also been closely associated with inflammation, responses to infection in the immune system and vascular integrity.

Platelets are normally in a quiescent state (inactive) in the blood circulation, until it is delicately triggered to response to sudden fluid shear stress and/or exposure to extracellular matrix (ECM) proteins on the walls of damaged vessels It is the immediate mechanical responsiveness of platelets towards activation and adhesion to sites of injury anjd the smallest changes or disturbances to regular laminar flow that makes it profoundly difficult to quantify in vivo $[1,2]$. There have been numerous in vitro systems designed to measure the adhesive and activating events of platelets during thrombus formation. However, , the innate mechanisms that control thrombus propagation, thrombus stability and embolism in an in vivo setting remains poorly quantified and understood.[3, 4].

\section{Imaging thrombus under flow using microfluidics}

The utilization of microfluidic devices for biological studies has developed into a popular field that allows clear ex vivo observation and measurement of thrombus formation, but still maintains the basic physiological environment by surface modification, shear stress control or different geometrical designs. In recent years, the development of microfluidic techniques has merged into a versatile tool for investigating platelet functions and roles of specific platelet receptor and response to different types of agonists, including collagen or shear conditions (references). Along with this, various kinds of flow assays have been proposed for fast and low cost diagnosis in clinical settings. Although the microfluidic device offers a highly flexible and stable platform for platelet and thrombosis studies, there are still insufficiencies and limitations. A challenge in this field is that the lack of standardization. Different microfluidic channels designs, materials used and devices that results in inconsistency. Furthermore, imaging instruments that can accurately quantify physical and molecular parameters of platelets during the thrombosis processes as well as the changes in the microenvironment with in the body would be a game changer in platelet biology. To better dissect the processes that imaging can quantify, we

*Corresponding author: steve.lee@ anu.edu.au 
separate the challenges into three broad imaging categories namely: morphological, molecular, and fluidic. Morphological information refers to accurate mapping of the physical dimension of a developing thrombus. Molecular imaging refers to individual platelets surface protein expression and signalling events involved at the different stages of activation. Finally, real time recording of fluidic flow patterns or shear rate manipulation over the developing thrombus would provide the ability to localise the effects of a shear gradient in situ.

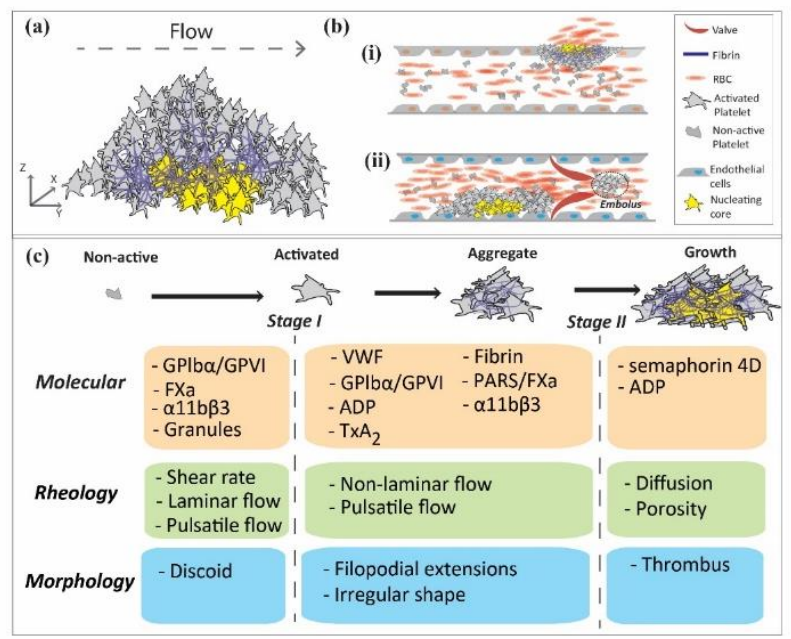

Figure 1. Shows the different molecular, rheology and morphological events that needs to be imaged under flow to study blood thrombus.

\section{Multimodality imaging in thrombus and platelet studies}

There are over half a million platelets in a single microliter of blood and each platelet are intricately crucial to the role of haemostasis; the ability to arrest blood loss after traumatic injury. There is crucial need to develop a fundamental understanding of how platelets respond to variations in changing shear gradient at a molecular and morphology level. Hence the simultaneous quantification of platelet thrombus formation and platelet receptor expression and shedding with precise determination of shear rates to micrometer levels across an individual thrombus, (on the same sample) will enable sheardependent effects on molecular events to be directly correlated. There are therefore enormous clinical and therapeutic implications for these shear-related events, and for the role of altered vascular rheology and shear in thrombus formation in diseased vessels. All in all, a comprehensive imaging system that can directly records the morphology, molecular and fluidic of a developing thrombus under flow will have a paradigm shift in platelet biology. An example of an effective use of multi-scale imaging for platelet studies was conducted by Gaertner and colleagues [5]. They have shown that platelet have an innate ability to detect invasion of foreign entities along the vascular bed using a series of imaging tools (fluorescence, FIB-SEM, multiphoton microscopy and differential interference contrast) to correlate the morphological and molecular from microscale to nanoscale. By gathering evidence through these imaging tools, they demonstrated that platelets have the strength to overcome adhesion forces and migrate towards invading bacteria. This extraordinary finding rests on the hypothesis that membrane adhesion receptors of adhering platelets can actively probe the mechanical properties of an adhesive substrate. Hence, the ability to combine combining two or more complementary imaging tools for platelets and thrombus studies could produce multiple viewpoints of a developing thrombus by simultaneously capturing biological activities at single platelet level and tracking the overall packing density of thrombus under flow. For example, it is easily conceivable for one to combine volumetric imaging techniques: quantitative phase microscopy and lightsheet to investigate the morphology of the thrombus and platelets volumetrically. This is especially because light sheet microscopy uses very low density of light and causes almost no adverse phototoxic outcomes. The lightsheet component of the microscopy system will be able to capture individual calcium signals of platelets interacting and at the same time a QPM [6] can directly quantifies the overall growth of the thrombus under continuous fluid flows. The simultaneous acquisition of these parameters will allow investigators to study the interplay between microrheological and agonist-dependent mechanisms of heterogeneous platelet activation within a developing thrombus with un-parallel clarity and resolution not previously obtained. While current optical imaging studies using multiphoton microscope in vivo [7] have shown evidence of heterogeneous molecular signatures between platelets within a single thrombus i.e. fully activated, the ability to quantify individual platelet interactions in a large thrombus at a sub-cellular level under flow is a grand challenge. In conclusion, the increasing use of advanced imaging techniques in platelet and thrombus studies would inevitable increase our understanding of thrombotic processes that occurs at short time intervals (milliseconds to seconds) on a micrometre size scale and with rapidly changing fluidic flows. We anticipate that in situ fluidic measurements combined with optical imaging techniques would be a necessary addition for the next generation of platelet imaging tools

\section{References}

1. Dreymueller, D., S. Uhlig, and A. Ludwig, Am J Physiol Lung Cell Mol Physiol, 2015. 308(4): p. L325-43.

2. Coenen, D.M., T.G. Mastenbroek, and J.M.E.M. Cosemans, Blood, 2017. 130(26): p. 2819-2828.

3. Tomaiuolo, M., L.F. Brass, and T.J. Stalker, Interv Cardiol Clin, 2017. 6(1): p. 1-12.

4. Jackson, S.P., Blood, 2007. 109(12): p. $5087-$ 5095.

5. Gaertner, F., et al., Cell, 2017. 171(6): p. 13681382.e23.

6. He, X., et al., Advanced Biosystems, 2018. 2(10): p. 1800089.

7. Li, Y., et al., Journal of Biophotonics, 2018. 11(7): p. e201700341. 